\title{
Identification of the mandibular landmarks in a pediatric population
}

\author{
Kenan Cantekin ${ }^{1}$, Ahmet-Ercan Sekerci ${ }^{2}$, Ozkan Miloglu ${ }^{3}$, Suleyman-Kutalmis Buyuk ${ }^{4}$
}

${ }^{1}$ DDS, PhD, Assistant Professor, Department of Paediatric Dentistry, Faculty of Dentistry, Erciyes University, Kayseri, Turkey

${ }^{2}$ DDS, PhD, Assistant Professor, Department of Maxillofacial Radiology, Faculty of Dentistry, Erciyes University, Kayseri, Turkey

${ }^{3}$ DDS, PhD, Assistant Professor, Department of Maxillofacial Radiology, Faculty of Dentistry, Ataturk University, Erzurum, Turkey

${ }^{4}$ DDS, Research Assistant, Department of Orthodontics, Faculty of Dentistry, Erciyes University, Kayseri, Turkey

Correspondence:

Department of Pediatric Dentistry

Faculty of Dentistry, Erciyes University

38039, Kayseri, Turkey

k_cantekin@hotmail.com

Cantekin K, Sekerci AE, Miloglu O, Buyuk SK. Identification of the mandibular landmarks in a pediatric population. Med Oral Patol Oral Cir Bucal. 2014 Mar 1;19 (2):e136-41.

http://www.medicinaoral.com/medoralfree01/v19i2/medoralv19i2p136.pdf

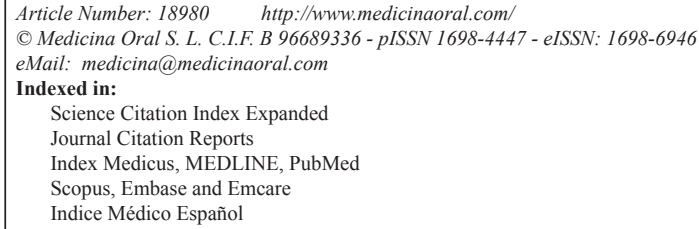

\begin{abstract}
Objectives: The aim of this study was to determine and compare the reliability to accomplish of common mandibular landmarks and to determine the incidence of incisive canals, anterior looping, and lingual foramina in children from panoramic and CBCT images.

Study Design: Panoramic and CBCT images from 100 children and adolescent patients were randomly selected. In order to grade the visibility of mandibular anatomical landmarks, a four-point rating scale was used.

Results: In panoramic images, the mandibular canal could be observed in $92.5 \%$ of cases, with good visibility in $12.0 \%$. The mental foramen could be observed in $44.5 \%$ of cases, while none had good visibility. Anterior looping of the mental nerve was present in $16.5 \%$ of the cases, and none had good visibility. An incisive canal could be identified in $22.5 \%$ of cases, with only $1.5 \%$ showing good visibility. The lingual foramen could be visualized in $61.0 \%$ of cases, with good visibility in $6 \%$. In CBCT images, the mandibular canal, the mental foramen, and the lingual foramen could be observed in $100 \%$ of the cases, with good visibility in $51.0 \%, 98.5 \%$, and $45.0 \%$ of cases, respectively. Anterior looping of the mental nerve was present in $26 \%$ of cases, with $2 \%$ having good visibility. An incisive canal could be identified in $49.5 \%$ of cases, with only $75 \%$ showing good visibility.

Conclusions: This study confirms the applicability of CBCT images to visualize critical structures in children.
\end{abstract}

Key words: Panoramic radiography, cone beam computed tomography, anatomical landmark.

\section{Introduction}

Today, panoramic radiography is often used for primary evaluations in dental practice to obtain information about the teeth, upper and lower jawbones, sinuses, temporomandibular joints, and other hard tissues of the head and neck. However, panoramic radiographies only give twodimensional information regarding the superimposition of all structures and lack information in a bucco-lingual direction. Contemporary imaging techniques such as cone beam-computed tomography (CBCT) may be par- 
ticularly suitable in the evaluation of jaws, as three-dimensional visualization and the high-resolution analysis of the entire mandible provide adequate information to localize anatomical structures (1-3).

So far, several reports have been presented to locate and measure mandibular anatomical landmarks in adults using different radiological techniques, as visualized on panoramic $(4-7)$ or CBCT images $(3,8,9)$. However, no studies have been done to determine and compare the visibility of mandibular landmarks in children. The aim of this study was to determine the reliability of using panoramic and CBCT images to identify and compare common mandibular landmarks and to determine the incidence of incisive canals, anterior looping, and lingual foramina in children.

\section{Material and Methods}

We defined five landmarks and collected data directly from mandibles and indirectly from panoramic and CBCT images of the mandibles in the same children. The anatomical landmarks in mandibles were the following: (1) the mandibular canal, (2) the mental foramen, (3) the anterior looping of the mental nerve, (4) the incisive canal, and (5) the lingual foramen.

Panoramic and CBCT images from 100 children and adolescent patients were randomly selected from existing records in the Department of Oral and Maxillofacial Radiology at the University of Erciyes, Kayseri, Turkey. All of the patients had been referred for CBCT diagnosis and treatment planning, consisting of 23 impacted teeth patients, 47 orthodontic patients, 19 possible pathosis patients, five supernumerary teeth patients, and six TMJ disorder patients (Table 1).

All radiographs were performed by x-ray technicians

Table 1. Description of the subjects and their indications for cone beam CT (CBCT).

\begin{tabular}{|l|c|}
\hline Age (years) & \\
\hline Gender (n) & $12.34 \pm 3.72$ \\
\hline Boy & 57 \\
\hline Girl & 43 \\
\hline Reason for Scan & No of Subjects \\
\hline Impaction localization & 23 \\
\hline Orthodontic records & 57 \\
\hline Other possible pathosis & 9 \\
\hline Supernumerary teeth localization & 5 \\
\hline TMJ assessment & 6 \\
\hline
\end{tabular}

who had a minimum of five years of work experience as of 1996, using an orthpantomography device (Planmeca Proline CC 2002, 60-80 kVp, 8-10 mA, 12.8 second exposure time, Helsinki, Finland) with a magnification factor of 1.2. The cone beam images were acquired using a Newton 5G (Quantitative Radiology, Verona, Italy) Flat panel-based CBCT machine. To establish a consistent orientation in the images, each patient was placed in a horizontal position such that the Frankfort horizontal plane (the plane between the highest point of the external auditory canal's opening and the orbit's lowest point) was perpendicular to the table, with the head within the circular gantry housing the x-ray tube. The $\mathrm{x}$-ray tube detector system performed a $360^{\circ}$ rotation around each patient's head, with a scanning time of $36 \mathrm{~s}$. The scanner operated with a maximum output of $110 \mathrm{KV}$ and $15 \mathrm{mAs}$, a $0.16-\mathrm{mm}$ voxel size and a typical exposure time of $5.4 \mathrm{~s}$. The QR-NNT software version 2.21 (Quantitative Radiology) was used to analyze the images. Approval from the ethics committee was not required for this retrospective study.

In order to grade the visibility of mandibular anatomical landmarks, the following four-point rating scale was used: No visibility $=$ important structures are not visualized Poor $=$ important structures are not diagnostic Moderate $=$ important structures are diagnostic but could be improved

Good = important structures are optimally visualized All images were scored by two well-trained dental specialists. Evaluated landmarks are denoted in figures 1,2 .
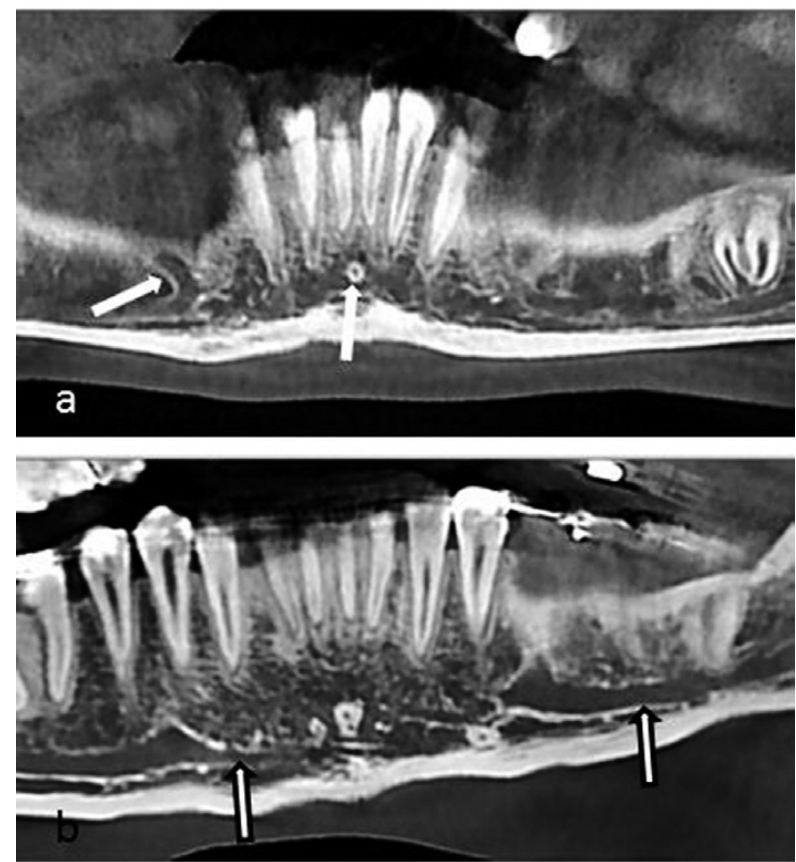

Fig. 1. Cropped cone beam computed tomographic panoramic view of a 17-year-old boy with denoted structures; anterior looping of the mental nerve (right side) and lingual foramen (a), and incisive canal (right side) and mandibular canal (left side) of a 16-year old a girl (b). 

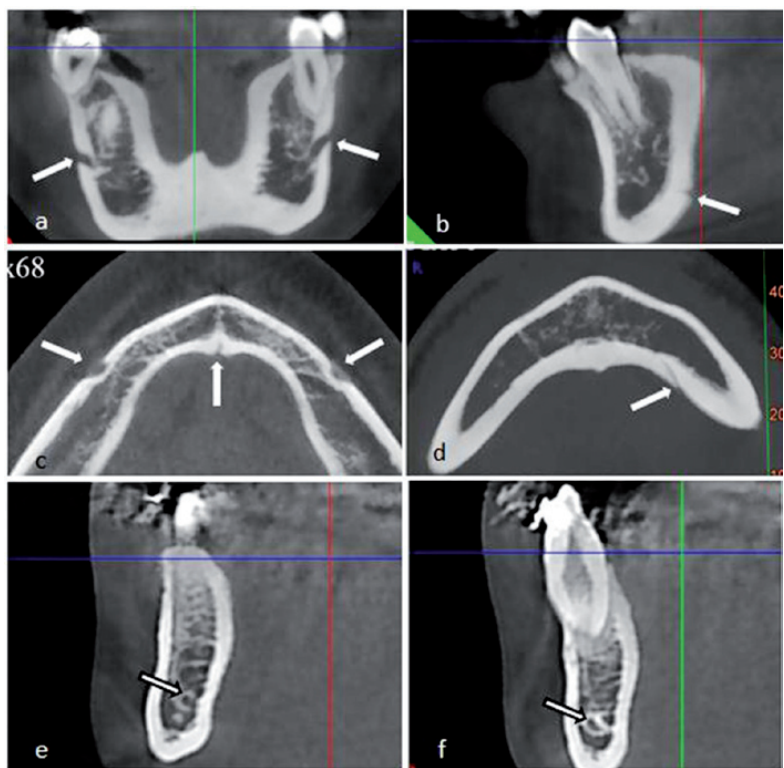

Fig. 2. Coronal reformatted cross-sectional image showing mental foramina of a 17-year old boy (a), lingual foramen (b). Axial view of a 14-year old a boy show inf mental and lingual foramina (c,d). Incisive canal (on right and left side of the interforaminal region) shown on reformatted cross-sectional images as a rounded radiolucent area surrounded by a radiopaque rim representing the canal walls (arrows) (e,f).

In the next step, selected cases were independently reevaluated by the two examiners to diagnose and classify the cases into different abnormality subtypes, such as congenital changes, malignant and benign tumors, odontogenic lesions, bone-related lesions, traumatic lesions (bony fractures), and inflammatory lesions (mucosal thickening, retention cysts, opacification, sinus polyps, and antroliths). Data were gathered and divergences between the examiners were solved by reaching a consensus.

-Statistical analyses

All calculations were processed using the Statistical Package for Social Science statistical software (version 16; SPSS Inc., Chicago, Illinois). Descriptive statistics including tables were used to display information. A chi-square test was used to compare the CBCT and panoramic images. Kappa statistics were also used to assess inter-examiner consistency.

\section{Results}

-Visibility rating of anatomical landmarks on panoramic radiographs

The visibility rating score in percentage of different anatomical landmarks, as illustrated on panoramic images, is shown in figures 3,4 . The mandibular canal could be observed in $92.5 \%$ of 100 cases, with good visibility in $12.0 \%$. The mental foramen could be observed in $44.5 \%$ of cases, while no cases had good visibility. Anterior looping of the mental nerve was present in $16.5 \%$ of cases, but no cases had good visibility. An incisive canal could be identified in $22.5 \%$ of cases, with only $1.5 \%$ showing good visibility. The lingual foramen could be visualized in $61.0 \%$ of cases, with good visibility in only $6 \%$.

-Visibility rating of anatomical landmarks on CBCT scan images

The visibility of anatomical landmarks on CBCT scan images is shown in figures 3,4. The mandibular canal, the mental foramen, and the lingual foramen could be observed in $100 \%$ of cases, with good visibility in

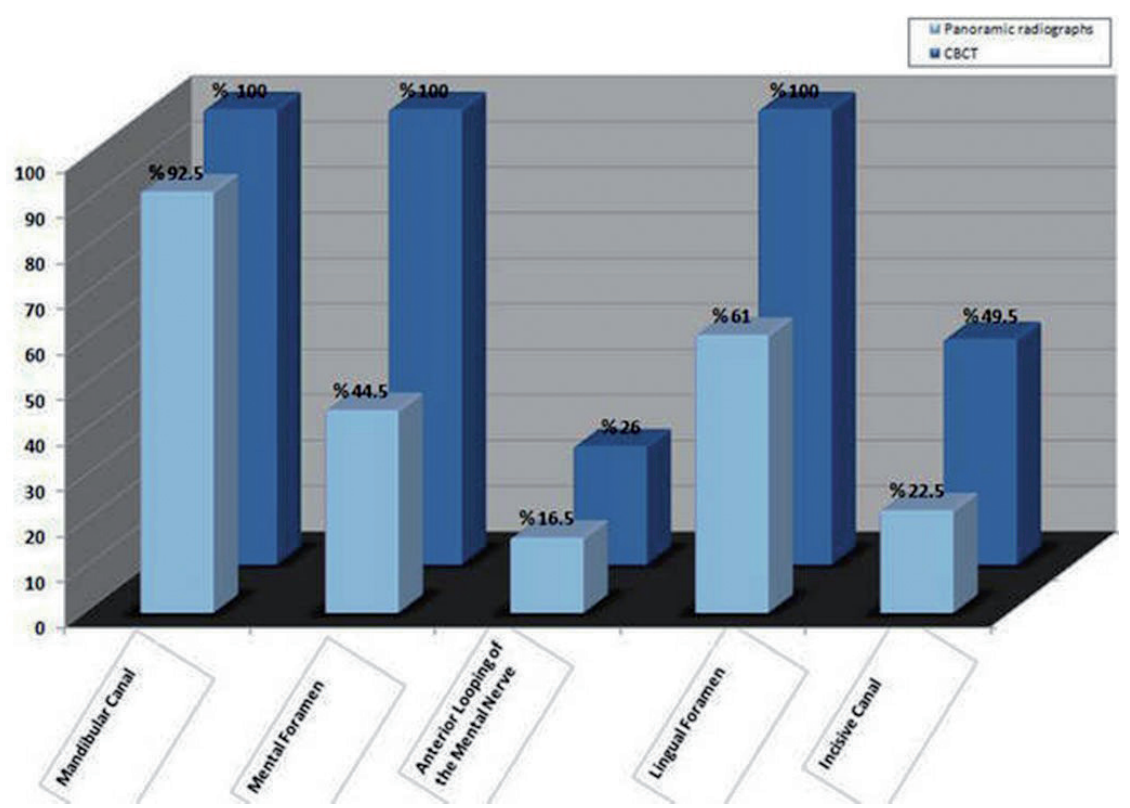

Fig. 3. Good visibility of anatomical structures in each group. 


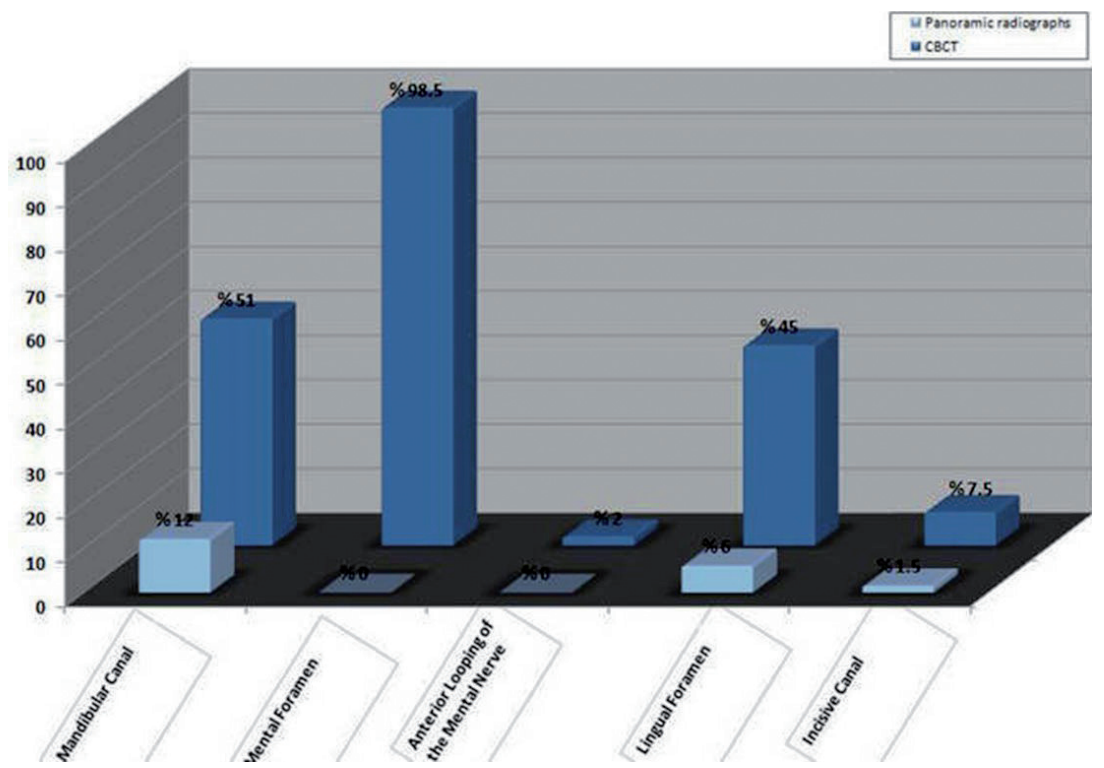

Fig. 4. Appearance of anatomical structures in each group.

$51.0 \%, 98.5 \%$, and $45.0 \%$ of cases, respectively. Anterior looping of the mental nerve was present in $26 \%$ of cases, with $2 \%$ having good visibility. An incisive canal could be identified in $49.5 \%$ of the cases, with only $7.5 \%$ showing good visibility.

Kappa statistics indicated excellent agreement for the observations of the anatomical landmarks as compared to the expert consensus statement. Kappa values for the panoramic images were $0.94,0.99,0.89,0.90$, and 0.86 for the mandibular canal, mental foramen, incisive canal, lingual foramen, and anterior looping, respectively. Additionally, Kappa values for the panoramic images were $1.00,1.00,0.93,1.00$, and 0.96 for the mandibular canal, mental foramen, incisive canal, lingual foramen, and anterior looping, respectively.

-Other findings

Table 2 represents all of the findings from the 100 scans.

\section{Discussion}

Several factors contribute to the reliability of landmark identification in children: the density and sharpness of images, the anatomic complexity and superimposition of hard and soft tissues, the definition of the landmark, and the training level or experience of the observers, especially for pediatric dentistry $(10,11)$.

The mandibular canal could be observed on panoramic and CT scans in $92.5 \%$ and $100 \%$ of cases, with good visibility in $12.0 \%$ and $50 \%$ of them, respectively. These findings are less promising than those from a previous study on panoramic images, where a canal was visible in $99 \%$ of cases and good visibility in $49 \%$; (2) however, in
Table 2. Summary of all the findings seen in the $100 \mathrm{CBCT}$ scans (57 boys and 43 girls).

\begin{tabular}{|l|c|}
\hline Temporomandibular joint & N \\
\hline Condylar fracture & 4 \\
\hline Osteophytes & 1 \\
\hline Bifid condyle & 1 \\
\hline Impacted teeth & $\mathbf{2 3}$ \\
\hline canines & 17 \\
\hline premolars & 6 \\
\hline Supernumerary teeth & $\mathbf{5}$ \\
\hline Lateral incisors & 3 \\
\hline premolars & 2 \\
\hline Other pathosis & $\mathbf{9}$ \\
\hline Residual cyst & $\mathbf{4}$ \\
\hline Nasopalatine canal cyst & $\mathbf{3}$ \\
\hline Cherubism & 36 \\
\hline Orthodontic records & 7 \\
\hline Localization of canines & \\
\hline Resorption of incisors after ectopic eruption of & 14 \\
\hline Obstructive sleep apnea & \\
\hline
\end{tabular}


accordance with prior studies on CT scans, a canal was visible in $97 \%$ and $99 \%$ of cases in present study $(3,12)$. Olivier (13) was the first to describe the course of the incisive nerve as a continuation of the inferior alveolar nerve traveling through a canal. Mardinger et al. (6) and Mwaiva et al. (7) anatomically observed an incisive canal in $80 \%$ and $96 \%$ of mandibles, respectively. Other studies, however, have neglected to identify the presence of a true incisive canal. $(14,15)$ The present anatomical study did not fully confirm the existence of the incisive canal, as it could only be seen in $49.5 \%$ (CT scan) and $22.5 \%$ (panoramic images) of the mandibles. The relatively low occurrence rate of the incisive canal in anatomical studies makes this observation most likely an anatomical variation.

In this study, mental foramina could be identified in $44.5 \%$ (panoramic images) and 100\% (CT scan) of cases, which is lower the findings of other studies on panoramic images $(3,12)$ and in accordance with CT scan studies (2).

An important anatomical variation in the interforaminal region is the anterior looping of the mental nerve that was present in 26\% (CT scan) and 16.5\% (panoramic images) of cases. This is in accordance with the results of Misch and Crawford (16) and Jacobs et al., (2) who reported radiographic visibility of anterior looping on panoramic images in $12 \%$ and $11 \%$ of cases, respectively. On panoramic radiographs, Ngeouw et al. (17) observed anterior looping in $40.2 \%$ of lower jaws. The value reported in the CT scan analysis done by Jacobs et al. (3) is somewhat lower (7\% of the cases). Generally, the radiographic visibility may differ, to some extent, from the anatomical observations. However, Kaya et al. (18) and Uchida et al. (19) reported prevalence rates of $34 \%$ and $71 \%$ of anterior looping using CT images, respectively. Bavitz et al. (20) noted anterior looping in $21 \%$ of the cadaver mandibles they investigated. Variations in the reported incidence of this anatomical variant may depend on the criteria used to define anterior looping and the degree of resorption of the investigated mandibles.

The visibility of the lingual foramen using various conventional radiographic techniques has been documented in several radiographic studies, $(2,4,6,7,21)$ in which the limitation of plain film and panoramic radiography in identifying these structures is documented. The results of this observational study demonstrate that the lingual foramen is visible in $61.0 \%$ (panoramic image) and $100 \%$ (CT scan) of cases. These findings are upper with results reported by several authors $(3,8,22)$, who found that the lingual foramen was visible in $82 \%$ to $89 \%$ of cases when using a CT scan. On the other hand, our lingual foramen imaging results from panoramic images are significantly weaker than those of Jacobs in which the lingual foramen was visible in $71 \%$ of cases using panoramic images (2). When comparing results of the present study to those of other studies, ours are inferior to those reported anatomically $(23,24)$ for a number of reasons, apart from image quality of the CBCT and panoramic equipment, including patient examination procedure limitations (e.g., subtle patient movement), degradation of the image quality due to soft tissue scattering radiation, the degree of corticalization of the canal wall, or combination of the above.

CBCT in dentistry has provided an imaging solution that has none of the projection errors associated with magnification and none of the superimposition problems associated with traditional panoramic imaging (25). In addition, CBCT has a wide range of tools such as $3 \mathrm{D}$ reconstructions in any direction to permit accurate identification of landmarks. Studies have reported excellent accuracy of 3D computed tomography (CT) (26,27). In our study, both panoramic (2D) and CBCT (3D) were used, the identification of landmarks reflected a real clinical situation, and discrepancies in landmark identification were likely.

CBCT has probably been one of the most revolutionary innovations in the field of dentistry in the past decade, and it provides a novel platform for imaging of maxillofacial area (28). It also provides clear and accurate images of structures, and therefore is extremely useful for assessing the bone component. As the resultant images displayed are often corrected for magnification, accurate measurements can be derived from the reformatted 3D data (29). Radiographs in 2D are insufficient, especially in complex cases like impacted teeth, supernumerary teeth, and orthognathic surgeries. CBCT images provide far more detailed information than conventional $2 \mathrm{D}$ radiographs and are user friendly. Soft tissues, the skull, the airway, and dentition can be observed and measured on CBCT images at a 1:1 ratio. In terms of its clinical significance, CBCT provides an excellent tool for accurate diagnosis, more predictable treatment planning, more efficient patient management and education, improved treatment outcome, and patient satisfaction. The radiation doses from CBCT are significantly lower than medical CT, but generally higher than conventional dental radiography (30). Recently, the Sedentexct working group proposed provisional evidence-based selection criteria with clinical indications as to when CBCT should be performed (30). CBCT should only be used when the clinical question cannot be answered by conventional radiography, and the field of view (FOV) should be limited to the region of interest (31). Ideally, CBCT equipment should be able to offer a choice of volume sizes to reduce patients' radiation exposure levels. A risk-benefit analysis must be performed on each individual patient when CBCT is being considered. In order to assess the risk of CBCT, the effective dose must first be calculated as well. 


\section{References}

1. Hofschneider U, Tepper G, Gahleitner A, Ulm C. Assessment of the blood supply to the mental region for reduction of bleeding complications during implant surgery in the interforaminal region. Int $\mathrm{J}$ Oral Maxillofac Implants. 1999;14:379-83.

2. Jacobs R, Mraiwa N, Van Steenberghe D, Sanderink G, Quirynen $\mathrm{M}$. Appearance of the mandibular incisive canal on panoramic radiographs. Surg Radiol Anat. 2004;26:329-33.

3. Jacobs R, Mraiwa N, vanSteenberghe D, Gijbels F, Quirynen M. Appearance, location, course, and morphology of the mandibular incisive canal: an assessment on spiral CT scan. Dentomaxillofac Radiol. 2002;31:322-7.

4. Lindh C, Petersson A, Klinge B. Visualisation of the mandibular canal by different radiographic techniques. Clin Oral Implants Res. 1992;3:90-7.

5. Lindh C, Petersson A, Klinge B. Measurements of distances related to the mandibular canal in radiographs. Clin Oral Implants Res. 1995;6:96-103.

6. Mardinger O, Chaushu G, Arensburg B, Taicher S, Kaffe I. Anatomic and radiologic course of the mandibular incisive canal. Surg Radiol Anat. 2000;22:157-61.

7. Mraiwa N, Jacobs R, Moerman P, Lambrichts I, van Steenberghe $\mathrm{D}$, Quirynen M. Presence and course of the incisive canal in the human mandibular interforaminal region: two-dimensional imaging versus anatomical observations. Surg Radiol Anat. 2003;25:416-23.

8. Makris N, Stamatakis H, Syriopoulos K, Tsiklakis K, van der Stelt PF. Evaluation of the visibility and the course of the mandibular incisive canal and the lingual foramen using cone-beam computed tomography. Clin Oral Implants Res. 2010;21:766-71.

9. Lagravere MO, Low C, Flores-Mir C, Chung R, Carey JP, Heo G et al. Intraexaminer and interexaminer reliabilities of landmark identification on digitized lateral cephalograms and formatted 3-dimensional cone-beam computerized tomography images. Am J Orthod Dentofacial Orthop. 2010;137:598-604.

10. McWilliam JS, Welander U. The effect of image quality on the identification of cephalometric landmarks. Angle Orthod. 1978;48:49-56.

11. Houston WJ, Maher RE, McElroy D, Sherriff M. Sources of error in measurements from cephalometric radiographs. Eur J Orthod. 1986;8:149-51.

12. Jalili MR, Emaeelinejad M, Bayat M, Aghdasi MM. Appearance of anatomical structures of mandible on panoramic radiographs in Iranian population. Acta Odontol Scand. 2012;70:384-9.

13. Olivier $\mathrm{E}$. The inferior dental nerve and its nerve in the adult. $\mathrm{Br}$ Dent J. 1928;49:356-8.

14. Haribhakti VV. The dentate adult human mandible: an anatomic basis for surgical decision making. Plast Reconstr Surg. 1996;97:53641.

15. Denissen HW, Veldhuis HA, van Faassen F. Implant placement in the atrophic mandible: an anatomic study. J Prosthet Dent. 1984;52:260-3.

16. Misch CE, Crawford EA. Predictable mandibular nerve location-a clinical zone of safety. Int J Oral Implantol. 1990;7:37-40.

17. Ngeow WC, Dionysius DD, Ishak H, Nambiar P. A radiographic study on the visualization of the anterior loop in dentate subjects of different age groups. J Oral Sci. 2009;51:231-7.

18. Kaya Y, Sencimen M, Sahin S, Okcu KM, Dogan N, Bahcecitapar M. Retrospective radiographic evaluation of the anterior loop of the mental nerve: comparison between panoramic radiography and spiral computerized tomography. Int J Oral Maxillofac Implants. 2008;23:919-25.

19. Uchida Y, Yamashita Y, Goto M, Hanihara T. Measurement of anterior loop length for the mandibular canal and diameter of the mandibular incisive canal to avoid nerve damage when installing endosseous implants in the interforaminal region. J Oral Maxillofac Surg. 2007;65:1772-9.

20. Bavitz JB, Harn SD, Hansen CA, Lang M. An anatomical study of mental neurovascular bundle-implant relationships. Int J Oral Maxillofac Implants. 1993;8:563-7.
21. Mardinger O, Chaushu G, Arensburg B, Taicher S, Kaffe I. Anterior loop of the mental canal: an anatomical-radiologic study. Implant Dent. 2000;9:120-5.

22. Tepper G, Hofschneider UB, Gahleitner A, Ulm C. Computed tomographic diagnosis and localization of bone canals in the mandibular interforaminal region for prevention of bleeding complications during implant surgery. Int J Oral Maxillofac Implants. 2001;16:6872 .

23. McDonnell D, Reza Nouri M, Todd ME. The mandibular lingual foramen: a consistent arterial foramen in the middle of the mandible. J Anat. 1994;184:363-9.

24. Liang X, Jacobs R, Lambrichts I, Vandewalle G. Lingual foramina on the mandibular midline revisited: a macroanatomical study. Clin Anat. 2007;20:246-51.

25. Waitzman AA, Posnick JC, Armstrong DC, Pron GE. Craniofacial skeletal measurements based on computed tomography: Part II. Normal values and growth trends. Cleft Palate Craniofac J. 1992;29:118-28.

26. Matteson SR, Bechtold W, Phillips C, Staab EV. A method for three-dimensional image reformation for quantitative cephalometric analysis. J Oral Maxillofac Surg. 1989;47:1053-61.

27. Tyndall DA, Renner JB, Phillips C, Matteson SR. Positional changes of the mandibular condyle assessed by three-dimensional computed tomography. J Oral Maxillofac Surg. 1992;50:1164-72.

28. Agrawal JM, Agrawal MS, Nanjannawar LG, Parushetti AD. CBCT in Orthodontics: The Wave of Future. J Contemp Dent Pract. 2008;14:153-7.

29. Angelopoulos C, Thomas SL, Hechler S, Parissis N, Hlavacek M. Comparison between digital panoramic radiography and cone-beam computed tomography for the identification of the mandibular canal as part of presurgical dental implant assessment. J Oral Maxillofac Surg. 2008;66:2130-5.

30. Ludlow JB, Ivanovic M. Comparative dosimetry of dental CBCT devices and 64-slice CT for oral and maxillofacial radiology. Oral Surg Oral Med Oral Pathol Oral Radiol Endod. 2008;106:106-14. 31. Isaacson KG, Thom AR, Horner K, Whaites E. Orthodontic Radiographs. Guidelines London. British Orthodontic Society. 2008. 